\title{
THERAPY
}

\section{Shot at sciatica relief misses the mark}

Etanercept delivered by epidural injection is no better at relieving pain than similarly administered steroids or saline, according to a randomized, controlled trial of 84 patients.

Steroid injection is widely used as a therapy for lumbosacral radiculopathy (commonly recognized as sciatica), but reports of the safety and efficacy of this approach are mixed. Evidence pointing to TNF as the root cause of the condition suggests that targeting this inflammatory cytokine might be more effective than treating general inflammation.

On the basis of animal studies and preliminary clinical data, a multicentre group of investigators chose to evaluate a small, locally applied dose of the antiTNF agent etanercept. The investigators expected that this approach would be effective and less likely to induce adverse effects than higher doses used systemically.

In the comparative-effectiveness study, patients were randomly allocated to receive $4 \mathrm{mg}$ etanercept, $60 \mathrm{mg}$ methylprednisolone acetate or normal saline administered by image-guided transforaminal injection, followed by a second injection 2 weeks later; all patients also received local anaesthetic ( $0.5 \mathrm{ml}$ of $0.5 \%$ bupivacaine). 1 month after the second injection, those patients who received steroids reported greater reductions in pain than those in the other groups; however, the improvements were modest and did not reach statistical significance.

Although the results are consistent with steroid injections having a moderate, short-term benefit, and with there being no advantage of epidural etanercept over saline, they are inconclusive. Future studies might look at the effectiveness of higher doses of etanercept, or longer-acting cytokine inhibitors.

\section{Sarah Onuora}

Original article Cohen, S. P. et al. Epidural steroids, etanercept, or saline in subacute sciatica: a multicenter, randomized trial. Ann. Intern. Med. 156, 551-559 (2012) 